Hautarzt 2016 $67: 768$

DOI 10.1007/s00105-016-3852-9

Online publiziert: 25 . Juli 2016

๑) Springer-Verlag Berlin Heidelberg 2016

CrossMark

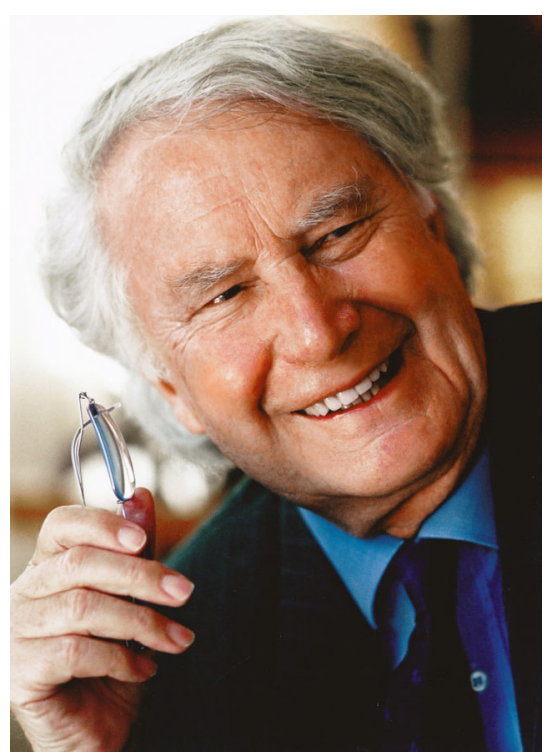

Abb. $1 \Delta$ Dr. Heinz Maurer, Gründer des Weltunternehmens Sebapharma

Im Alter von 94 Jahren ist Herr Dr. med. Heinz Maurer am 22.4.2016 in Boppard verstorben (• Abb. 1). Mit ihm hat die Dermatologie einen Forscher und Entwickler verloren, dessen Lebenswerk der Etablierung sowie der wissenschaftlichen Untermauerung der physiologischen Hautreinigung und -pflege mit pH 5,5 galt.

\section{Firmengründer und Pionier der medizinischen Hautpflege}

Als Assistent an der Bonner Universitätsklinik kam Dr. Heinz Maurer neben der inneren Medizin intensiv mit der Dermatologie in Kontakt. Aufgerüttelt durch das damals noch geltende Waschverbot bei Ekzempatienten etablierte er aufgrund guter Kontakte zur Pädiatrie die Hautreinigung mit sauren seifenfreien Waschstücken (Syndets) auch als Therapiebegleitung bei der Windeldermatitis. Damit bahnte er einen inzwischen allgemein anerkannten Weg.

\author{
W. Gehring \\ Bietigheim, Deutschland
}

\title{
Nachruf auf Dr. Heinz Maurer
}

1967 gründete der Mediziner die Sebamat-Chemie GmbH und ermöglichte durch die Einführung des sauren seifenfreien Syndets sebamed die Aufhebung des Waschverbots bei vielen Hautkrankheiten. Die Sebapharma GmbH \& Co. KG wurde von ihm 1983 gegründet. Sein unermüdlicher Drang nach Forschung und Entwicklung neuer Präparate hat dieser Firma in Deutschland national und international zum Durchbruch verholfen. 1992 rief Dr. Maurer den Wissenschaftlichen Beirat der Firma ins Leben, dem seitdem namhafte dermatologische und pharmazeutische Wissenschaftler angehören.

\section{Förderer und Unterstützer der Dermatologie}

Die Dermatologie verdankt Herrn Dr. Maurer viel. 1995 wurde der Heinz Maurer-Preis ausgelobt und 1996 erstmals verliehen. Im Rhythmus von zwei Jahren werden hervorragende wissenschaftliche Arbeiten zum Thema „Hautbarriere und Umwelt“ prämiert. Dabei finden Untersuchungen zur Grundlagenforschung und klinische Studien gleichermaßen Berücksichtigung.

Auf seine Initiative gehen die Symposien in Bad Griesbach (1988) „Hautreinigung mit Syndets“ und in Dessau (1997) "Nutzen und Risiko von Kosmetika“ zurück. Anlässlich der 10. Verleihung des Heinz Maurer-Preises fand 2014 in Boppard ein internationales Meeting zum Thema „Review and Preview in Science for Healthy Skin“ statt.

Durch seine Mitgliedschaften in der Deutschen Dermatologischen Gesellschaft, in der Arbeitsgemeinschaft Aesthetische Dermatologie sowie der Gesellschaft für Dermopharmazie war Dr. Heinz Maurer der Dermatologie nahe, hat diese gefördert und unterstützt.
Ganz besonders muss sein soziales Engagement hervorgehoben werden. Dr. Heinz Maurer hat die deutsche Hautkrebsforschung durch eine großzügige Spende gefördert und soziale Projekte in Chile, Altenheime, SOS-Kinderdörfer, den Hermann-Gmeiner-Fonds und Cap Anamur finanziell unterstützt. Sein persönlicher Einsatz fand vielfache Anerkennung und Würdigung. Ihm wurden das große Stadtsiegel der Stadt Boppard, das Bundesverdienstkreuz, die „Bene merenti“-Medaille der Universität Regensburg und der Verdienstorden des Landes Rheinland-Pfalz verliehen.

Herr Dr. Heinz Maurer ist nach einem erfüllten Leben gegangen. Sein unermüdlicher Forschungs- und Entwicklungsdrang hat die moderne Hautreinigung und Hautpflege geprägt und macht seinen Namen unvergesslich.

\section{Korrespondenzadresse}

\section{Prof. Dr. W. Gehring}

Willi-Schertle-Ring 14, 76467 Bietigheim, Deutschland

W.M.Gehring@t-online.de

Prof. Dr. W. Gehring ist Ehrenmitglied des Wissenschaftlichen Beirates bei Sebapharma und Vorsitzender der Jury des Heinz Maurer Preises. 